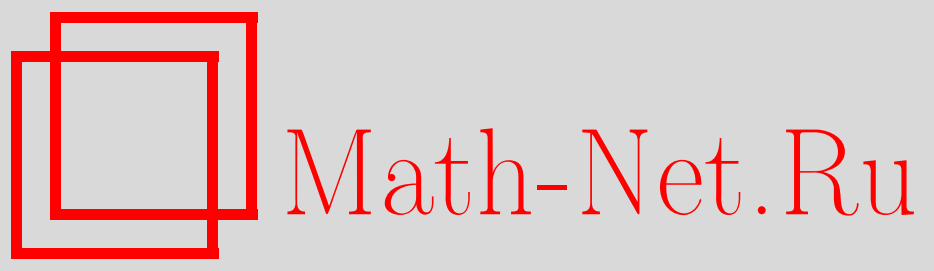

С. Ю. Лукащук, О построении законов сохранения для интегро-дифференциальных уравнений дробного порядка, ТМФ, 2015, том 184, номер 2, 179-199

DOI: https://doi.org/10.4213/tmf8833

Использование Общероссийского математического портала Math-Net.Ru подразумевает, что вы прочитали и согласны с пользовательским соглашением http://www.mathnet.ru/rus/agreement

Параметры загрузки:

IP : 52.23 .180 .231

26 апреля 2023 г., 07:43:14

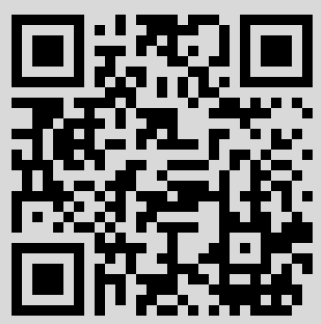




\title{
ФИЗИКА
}

Том 184, № 2

август, 2015

(C) 2015 г.

С. Ю. Лукащук*

\section{О ПОСТРОЕНИИ ЗАКОНОВ СОХРАНЕНИЯ ДЛЯ ИНТЕГРО-ДИФФЕРЕНЦИАЛЬНЫХ УРАВНЕНИЙ ДРОБНОГО ПОРЯДКА}

\begin{abstract}
В классе функций, зависящих от линейных интегро-дифференциальных переменных дробного порядка, доказан аналог фундаментального операторного тождества, связывающего инфинитезимальный оператор группы точечных преобразований, дифференциальный оператор Эйлера-Лагранжа и операторы Нётер. С использованием этого тождества доказаны дробно-дифференциальные аналоги теоремы Нётер и ее обобщения, применимые к уравнениям с интегралами и производными дробного порядка различных видов, являющимся уравнениями Эйлера-Лагранжа. В явном виде приводятся дробно-дифференциальные обобщения операторов Нётер, что дает для уравнений рассматриваемого вида эффективный способ построения законов сохранения, который иллюстрируется тремя примерами.
\end{abstract}

Ключевые слова: интегро-дифференциальное уравнение дробного порядка, симметрия, закон сохранения, фундаментальное операторное тождество, теорема Нётер.

DOI: $10.4213 / \operatorname{tmf} 8833$

\section{1. ВВЕДЕНИЕ}

В последние годы теория интегро-дифференцирования дробного порядка [1], [2] все активнее используется как мощный математический аппарат исследования физических процессов, протекающих в неоднородных сложных средах с эффектами степенной памяти и пространственной нелокальности. Уравнения с интегралами и производными дробных порядков различных типов успешно используются для описания процессов аномального переноса (таких, как субдиффузия, супердиффузия, диффузионно-волновой перенос и др.), турбулентности, фрактальной динамики классических и квантовых систем, моделирования реологических сред [3]-[6]. Однако методы исследования таких уравнений, особенно нелинейных, разработаны еще крайне недостаточно.

*ФГБОУ ВПО «Уфимский государственный авиационный технический университет», Уфа, Россия. Е-mail: lsu@mail.rb.ru 
Одним из эффективных подходов к исследованию и построению решений нелинейных дифференциальных уравнений является групповой анализ [7], [8]. В работах [9]-[11] базовые методы группового анализа были успешно распространены на дифференциальные уравнения с производными дробного порядка различных типов (так называемые дробно-дифференциальные уравнения). Там же были найдены симметрии и построены инвариантные решения ряда таких уравнений.

Со времени знаменитой работы Нётер [12] хорошо известна связь симметрий с законами сохранения, играющими фундаментальную роль в современной физике. В работе [13] было доказано обобщение теоремы Нётер, дающее не только необходимое, но и достаточное условие существования законов сохранения. На основе фундаментального операторного тождества, связывающего инфинитезимальный оператор группы преобразований, дифференциальный оператор Эйлера-Лагранжа и операторы Нётер, был разработан простой и эффективный конструктивный алгоритм построения законов сохранения с использованием симметрий для дифференциальных уравнений, имеющих лагранжиан (см., например, монографии [7], [8]).

Для дифференциальных уравнений дробного порядка в настоящее время используется несколько определений законов сохранения. Так, в работах [14]-[17] используется классическое определение, в котором под законом сохранения понимается равенство нулю дивергенции некоторого вектора (называемого сохраняющимся). Такое определение является естественным многомерным обобщением понятия первого интеграла для обыкновенных дифференциальных уравнений, которое широко применяется и в теории интегро-дифференцирования дробного порядка. Именно такое классическое определение закона сохранения будет использоваться в настоящей работе.

В работах [18]-[20] применяется формальное обобщение классического определения, в котором все или некоторые частные производные первого порядка, входящие в оператор дивергенции, заменены на частные производные дробного порядка типа Капуто или Римана-Лиувилля по соответствующим переменным. Однако нетрудно показать, что такое определение сводится к классическому (как это сделано, например, в статье [18]). В работе [21] предложено дробно-дифференциальное обобщение понятия закона сохранения, под которым понимается равенство нулю функциональной линейной комбинации левосторонней и правосторонней производных дробного порядка типа Римана-Лиувилля. Произведения функций, входящих в эту линейную комбинацию, являются компонентами сохраняющегося вектора. Такой подход несколько упрощает процедуру доказательства дробно-дифференциальных обобщений теоремы Нётер (см., например, работы [21]-[23]), однако физический смысл получающихся в результате сохраняющихся величин остается неясным. Интересно отметить, что в более новых работах [16], [17] авторы данного подхода вернулись к классическому определению закона сохранения.

В работах [15]-[17], [21]-[23] доказано несколько дробно-дифференциальных обобщений теоремы Нётер. Однако полученные в этих работах результаты применимы лишь для рассмотренных там лагранжианов некоторых частных видов. Так, в статьях [15], [23] рассматриваются лагранжианы, зависящие от одной независимой и одной зависимой переменной, а также от одной левосторонней производной типа Римана-Лиувилля дробного порядка $\alpha \in(0,1)$ от зависимой переменной. В работах [16] и [21] рассматриваются лагранжианы аналогичного вида, в которых до- 
бавлена зависимость соответственно от обычной первой производной зависимой переменной и от правосторонней производной типа Римана-Лиувилля. В статье [22] результаты работы [21] обобщаются на многомерный случай, когда лагранжиан зависит от левосторонних дробных производных типа Римана-Лиувилля своего дробного порядка по каждой независимой переменной. В результате складывается такая ситуация, что рассмотрение любого лагранжиана, не подпадающего под уже изученные в существующих работах частные случаи, приводит к необходимости заново проводить полное доказательство всех утверждений и получать новые формулы для построения соответствующих законов сохранения.

Предлагаемый в настоящей работе подход носит более общий характер, поскольку позволяет рассматривать лагранжианы, зависящие от произвольного количества интегро-дифференциальных переменных дробного порядка в $n$-мерном пространстве. При этом в качестве таких переменных могут выступать любые переменные, образованные путем применения к зависимой переменной произвольной суперпозиции операторов дифференцирования, левостороннего и правостороннего интегрирования любых дробных порядков $\alpha \in(0,1)$, действующих по любым независимым переменным. Лагранжианы такого вида включают в себя как частные случаи все виды лагранжианов, рассмотренных в работах [15]-[17], [21]-[23]. В настоящей работе доказывается справедливость дробно-дифференциального аналога фундаментального операторного тождества, с использованием которого далее доказываются дробнодифференциальные аналоги теоремы Нётер и ее обобщения, применимые к интегро-дифференциальным уравнениям дробного порядка, являющимся уравнениями Эйлера-Лагранжа с лагранжианами рассматриваемого типа. В явном виде строятся дробно-дифференциальные обобщения операторов Нётер. В результате задача нахождения законов сохранения для уравнений рассматриваемого вида по известным симметриям сводится к формальной и алгоритмизуемой процедуре применения этих операторов к соответствующему лагранжиану. Эффективнность предлагаемого подхода иллюстрируется тремя примерами.

\section{2. НЕОБХОДИМЫЕ ПОНЯТИЯ И ОПРЕДЕЛЕНИЯ}

2.1. Интегралы и производные дробного порядка. В настоящей работе будут использоваться следующие определения интегралов и производных дробного порядка [1], [2].

ОПРЕДЕЛЕНИЕ 1. Пусть $\varphi(x) \in L_{1}(a, b)$ (здесь $-\infty<a<b<\infty$ ). Интегралы

$$
\begin{aligned}
& { }_{a} I_{x}^{\alpha} \varphi(x) \equiv \frac{1}{\Gamma(\alpha)} \int_{a}^{x} \frac{\varphi(t)}{(x-t)^{1-\alpha}} d t, \quad x>a, \\
& { }_{x} I_{b}^{\alpha} \varphi(x) \equiv \frac{1}{\Gamma(\alpha)} \int_{x}^{b} \frac{\varphi(t)}{(t-x)^{1-\alpha}} d t, \quad x<b,
\end{aligned}
$$

называются соответственно левосторонним и правосторонним интегралами дробного порядка $\alpha>0$ типа Римана-Лиувилля.

С помощью операторов $(2.1),(2.2)$ и оператора дифференцирования $D_{x}$ определяются производные дробного порядка типа Римана-Лиувилля и Капуто. 
ОПРЕДЕЛЕНИЕ 2. Левосторонней и правосторонней производнъми дробного порядка $\alpha>0$ muпа Римана-Лиувилля от функции $\varphi(x)$, определенной на отрезке $[a, b]$, называются соответственно

$$
\begin{aligned}
& { }_{a} D_{x}^{\alpha} \varphi(x) \equiv D_{x}^{m}\left({ }_{a} I_{x}^{m-\alpha} \varphi(x)\right)=\frac{1}{\Gamma(m-\alpha)}\left(\frac{d}{d x}\right)^{m} \int_{a}^{x} \frac{\varphi(t)}{(x-t)^{\alpha-m+1}} d t, \\
& { }_{x} D_{b}^{\alpha} \varphi(x) \equiv(-1)^{m} D_{x}^{m}\left({ }_{x} I_{b}^{m-\alpha} \varphi(x)\right)=\frac{(-1)^{m}}{\Gamma(m-\alpha)}\left(\frac{d}{d x}\right)^{m} \int_{x}^{b} \frac{\varphi(t)}{(t-x)^{\alpha-m+1}} d t,
\end{aligned}
$$

где $m=[\alpha]+1$.

ОПРЕДЕЛЕНИЕ 3. Левосторонней и правосторонней производнъми дробного порядка $\alpha>0$ muna Kanymo от функции $\varphi(x)$, определенной на отрезке $[a, b]$, называются соответственно

$$
\begin{aligned}
& { }_{a}^{\mathrm{C}} D_{x}^{\alpha} \varphi(x) \equiv{ }_{a} I_{x}^{m-\alpha}\left(D_{x}^{m} \varphi(x)\right)=\frac{1}{\Gamma(m-\alpha)} \int_{a}^{x} \frac{\varphi^{(m)}(t)}{(x-t)^{\alpha-m+1}} d t, \\
& { }_{x}^{\mathrm{C}} D_{b}^{\alpha} \varphi(x) \equiv(-1)^{m}{ }_{x} I_{b}^{m-\alpha}\left(D_{x}^{m} \varphi(x)\right)=\frac{(-1)^{m}}{\Gamma(m-\alpha)} \int_{x}^{b} \frac{\varphi^{(m)}(t)}{(t-x)^{\alpha-m+1}} d t,
\end{aligned}
$$

где $m=[\alpha]+1$.

Отметим, что в настоящее время дробные производные (2.3)-(2.6) наиболее часто используются для построения различных математических моделей, учитывающих эффекты степенной памяти и пространственной нелокальности.

Следуя книге [1], обозначим через $A C^{m}[a, b]$ класс функций $f(x)$, непрерывно дифференцируемых на отрезке $[a, b]$ вплоть до порядка $m-1$ и таких, что производная $f^{(m-1)}(x)$ является абсолютно непрерывной функцией: $f^{(m-1)}(x) \in A C[a, b]$. Принадлежность функции $\varphi(x)$ классу $A C^{m}[a, b]$ является достаточным условием существования дробных производных типа Римана-Лиувилля (2.3), (2.4) и Капуто $(2.5),(2.6)[1],[2]$.

Приведенные определения дробных производных естественным образом обобщаются для функций многих переменных на частные производные дробного порядка путем замены оператора дифференцирования $D_{x}$ на операторы частного дифференцирования по соответствующей независимой переменной [1], [2].

2.2. Обобщение понятия дифференциальной функции. В групповом анализе дифференциальных уравнений используются понятия дифференциальных переменных и дифференциальных функций [8], [24].

Пусть $x=\left\{x^{i}\right\}(i=1,2, \ldots, n)$ - вектор независимых переменных и $u=u(x)-$ зависимая переменная.

ОПРЕДЕЛЕНИЕ 4. Дифференииальными переменными называются переменные

$$
u_{(1)}=\left\{u_{i_{1}}\right\}, \quad u_{(2)}=\left\{u_{i_{1} i_{2}}\right\}, \quad \ldots, \quad u_{(k)}=\left\{u_{i_{1} i_{2} \ldots i_{k}}\right\}, \ldots,
$$

определяющиеся системой соотношений

$$
\begin{gathered}
u_{i_{1}}=D_{i_{1}}(u), \quad u_{i_{1} i_{2}}=D_{i_{2}}\left(u_{i_{1}}\right)=D_{i_{2}} D_{i_{1}}(u), \quad \ldots, \\
u_{i_{1} i_{2} \ldots i_{k}}=D_{i_{k}}\left(u_{i_{1} i_{2} \ldots i_{k-1}}\right)=D_{i_{k}} D_{i_{k-1}} \ldots D_{i_{1}}(u)
\end{gathered}
$$

(здесь $\left.i_{1}, i_{2}, \ldots, i_{k}=1,2, \ldots, n\right)$. 
В этом определении и далее через $D_{i}$ обозначается оператор полного дифференцирования по переменной $x^{i}$.

ОПРЕДЕЛЕНИЕ 5 [24]. Дифференциальной функцией называется аналитическая функция конечного числа переменных $x, u, u_{(1)}, \ldots$ Максимальный порядок $k$ производной, входящей в дифференциальную функцию

$$
F=F\left(x, u, u_{(1)}, u_{(2)}, \ldots, u_{(k)}\right), \quad k<\infty
$$

называется порядком этой функции. Множество всех дифференциальных функций конечного порядка образует пространство дифференциальных функций и обозначается как $\mathcal{A}$.

Равенство $F\left(x, u, u_{(1)}, u_{(2)}, \ldots, u_{(k)}\right)=0$ (для $F \in \mathcal{A}$ ) определяет дифференциальное уравнение (в общем случае в частных производных) $k$-го порядка.

Попытка обобщить понятие дифференциальной функции на случай дифференциальных уравнений с производными дробного порядка типа Римана-Лиувилля или Капуто наталкивается на ряд принципиальных трудностей. Применение оператора дифференцирования к дифференциальной функции $k$-го порядка $(2.8)$ снова дает дифференциальную функцию конечного $(k+1)$-го порядка. Данное свойство перестает выполняться при замене оператора дифференцирования целого порядка на оператор дробного дифференцирования. Это обусловлено тем, что в отличие от оператора дифференцирования целого порядка операторы дробного дифференцирования являются нелокальными и в общем случае не коммутируют друг с другом [1], [2]. Кроме того, дробная производная от произведения двух функций представляется в виде бесконечного ряда (обобщенное правило Лейбница) [1], [2], а производная сложной функции - в виде четырехкратного ряда [9]. Тем не менее в подавляющем большинстве дифференциальных уравнений дробного порядка, использующихся в настоящее время на практике, неизвестная функция входит в операторы дробного интегро-дифференцирования линейно. Поэтому в первом приближении можно ограничиться рассмотрением функций, зависящих именно от таких линейных интегро-дифференциальных переменных.

Пусть $\Omega$ есть $n$-мерный параллелепипед в $\mathbb{R}^{n}$ :

$$
\Omega=\left[a^{1}, b^{1}\right] \times\left[a^{2}, b^{2}\right] \times \cdots \times\left[a^{n}, b^{n}\right], \quad-\infty<a^{i}<b^{i}<\infty \quad(i=1,2, \ldots, n) .
$$

Мы будем рассматривать операторы трех типов, действующие независимо по каждой координате $x^{i}(i=1,2, \ldots, n)$ вектора независимых переменных $x \in \Omega$, а именно операторы дифференцирования $D_{i}$, операторы левостороннего дробного интегрирования $I_{i+}^{\alpha_{i}} \equiv{ }_{a^{i}} I_{x^{i}}^{\alpha_{i}}$ порядка $\alpha_{i} \in(0,1)$ и операторы правостороннего дробного интегрирования $I_{i-}^{\beta_{i}} \equiv{ }_{x^{i}} I_{b^{i}}^{\beta_{i}}$ порядка $\beta_{i} \in(0,1)$. Объединим эти операторы в $2 n$ параметрическую матрицу

$$
B=\left(\begin{array}{cccc}
D_{1} & D_{2} & \ldots & D_{n} \\
I_{1}^{\alpha_{1}} & I_{2+}^{\alpha_{2}} & \ldots & I_{n+}^{\alpha_{n}} \\
I_{1-}^{\beta_{1}} & I_{2-}^{\beta_{2}} & \ldots & I_{n-}^{\beta_{n}}
\end{array}\right), \quad \alpha_{i}, \beta_{i} \in(0,1) \quad(i=1,2, \ldots, n)
$$


элементы которой будем обозначать как

$$
B_{i}^{\delta}= \begin{cases}I_{i+}^{\alpha_{i}}, & \delta=+1, \\ D_{i}, & \delta=0, \\ I_{i-}^{\beta_{i}}, & \delta=-1 .\end{cases}
$$

Обобщим понятие дифференциальных переменных (2.7) на случай интегро-дифференциальных операторов дробного порядка.

ОПРЕДЕЛЕНИЕ 6 . Переменные $u_{[k]}$, полученные в результате последовательного применения операторов (2.10) к зависимой переменной $u$ и определяемые соотношениями

$$
\begin{aligned}
u_{[1]}= & \left\{u_{i_{1}}^{\delta_{1}}\right\} \equiv\left\{B_{i_{1}}^{\delta_{1}}(u)\right\} \\
u_{[2]}= & \left\{u_{i_{1} i_{2}}^{\delta_{1} \delta_{2}}\right\} \equiv\left\{B_{i_{2}}^{\delta_{2}}\left(u_{i_{1}}^{\delta_{1}}\right)\right\}=\left\{B_{i_{2}}^{\delta_{2}} B_{i_{1}}^{\delta_{1}}(u)\right\}, \ldots, \\
u_{[k]}=\left\{u_{i_{1} i_{2} \ldots i_{k}}^{\delta_{1} \delta_{2} \ldots \delta_{k}}\right\} \equiv\left\{B_{i_{k}}^{\delta_{k}}\left(u_{i_{1} i_{2} \ldots i_{k-1}}^{\delta_{1} \delta_{2} \ldots \delta_{k-1}}\right)\right\}=\left\{B_{i_{k}}^{\delta_{k}} B_{i_{k-1}}^{\delta_{k-1}} \ldots B_{i_{1}}^{\delta_{1}}(u)\right\}, \ldots & \ldots \\
& \left(i_{1}, i_{2}, \ldots, i_{k}=1,2, \ldots, n, \quad \delta_{1}, \delta_{2}, \ldots, \delta_{k}=-1,0,1\right),
\end{aligned}
$$

будем называть линейными дробно-интегро-дифберенииалъными переменными.

Нетрудно заметить, что множество переменных $u_{[k]}$ включает в себя множество переменных $u_{(k)}$.

Обобщим теперь понятие дифференциальной функции.

ОпРЕДЕЛЕНиЕ 7. Аналитическая функция, зависящая от конечного числа переменных $x, u, u_{[1]}, \ldots$, называется линейно-дробно-интегро-дифференциальной функизей. Максимальный порядок $k$ переменных вида (2.11), входящих в дифференциальную функцию

$$
F=F\left(x, u, u_{[1]}, u_{[2]}, \ldots, u_{[k]}\right), \quad k<\infty,
$$

называется порядком этой функции. Множество всех линейно-дробно-интегро-дифференциальных функций конечного порядка обозначается как $\mathcal{F}$.

Заметим, что для определения функции вида (2.12) $k$-го порядка в общем случае требуется задание $(2 n)^{k}$ порядков дробного интегрирования.

ЗАМЕчАниЕ 1. Для существования линейно-дробно-интегро-дифференциальной функции $k$-го порядка $(2.12)$ в общем случае достаточно, чтобы $u(x) \in A C^{k}(\Omega)$. Однако для некоторых конкретных частных видов функций вида (2.12) это требование может быть ослаблено.

Равенство

$$
F\left(x, u, u_{[1]}, u_{[2]}, \ldots, u_{[k]}\right)=0
$$

определяет уравнение с интегро-дифференциальными операторами как дробных, так и целых порядков. Как частные случаи уравнение (2.13) включает в себя классические дифференциальные уравнения, интегральные уравнения дробных порядков, дробно-дифференциальные уравнения с производными типа Римана-Лиувилля 
и Капуто, а также целый ряд других известных классов интегро-дифференциальных уравнений.

Нетрудно показать, что если $F \in \mathcal{F}$, то в силу $(2.11) D_{i}(F) \in \mathcal{F}(i=1,2, \ldots, n)$. Действительно, оператор полного дифференцирования по переменной $x^{i}$ имеет вид

$$
D_{i}=\frac{\partial}{\partial x^{i}}+u_{i} \frac{\partial}{\partial u}+\sum_{s=1}^{k} D_{i}\left(u_{j_{1} \ldots j_{s}}^{\delta_{1} \ldots \delta_{s}}\right) \frac{\partial}{\partial u_{j_{1} \ldots j_{s}}^{\delta_{1} \ldots \delta_{s}}}
$$

и для функции (2.12) порядка $k$ мы получаем

$$
D_{i}(F)=\frac{\partial F}{\partial x^{i}}+u_{i} \frac{\partial F}{\partial u}+\sum_{s=1}^{k} D_{i}\left(u_{j_{1} \ldots j_{s}}^{\delta_{1} \ldots \delta_{s}}\right) \frac{\partial F}{\partial u_{j_{1} \ldots j_{s}}^{\delta_{1} \ldots \delta_{s}}}, \quad F \in \mathcal{F} .
$$

Частная производная функции $F$ по любой ее переменной принадлежит $\mathcal{F}$, а

$$
D_{i}\left(u_{j_{1} \ldots j_{s}}^{\delta_{1} \ldots \delta_{s}}\right)=u_{j_{1} \ldots j_{s} i}^{\delta_{1} \ldots \delta_{s} 0}
$$

поскольку $D_{i}=B_{i}^{0}$ в силу (2.10). В результате получаем, что функция $D_{i}(F) \in \mathcal{F}$ и имеет порядок $k+1$.

В дальнейшем будут также использоваться операторы, сопряженные к операторам (2.10) на $\Omega$. Так как

$$
\begin{gathered}
D_{i}^{*}=-D_{i}, \\
\left(I_{i+}^{\alpha}\right)^{*} \equiv\left({ }_{a^{i}} I_{x^{i}}^{\alpha}\right)^{*}={ }_{x^{i}} I_{b^{i}}^{\alpha} \equiv I_{i-}^{\alpha}, \\
\left(I_{i-}^{\beta}\right)^{*} \equiv\left({ }_{x^{i}} I_{b^{i}}^{\beta}\right)^{*}={ }_{a^{i}} I_{b^{i}}^{\beta} \equiv I_{i+}^{\beta},
\end{gathered}
$$

мы имеем

$$
\left(B_{i}^{\delta}\right)^{*}=\left\{\begin{aligned}
I_{i-}^{\alpha_{i}}, & \delta=+1, \\
-D_{i}, & \delta=0, \\
I_{i+}^{\beta_{i}}, & \delta=-1 .
\end{aligned}\right.
$$

Кроме того, определим оператор $J_{(i)}^{\delta}$, действующий на упорядоченную пару функций $\{f(x), g(x)\}(x \in \Omega)$ по следующему правилу:

$$
J_{(i)}^{\delta}\{f, g\}= \begin{cases}-\frac{1}{\Gamma\left(\alpha_{i}\right)} \int_{a^{i}}^{x^{i}} \int_{x^{i}}^{b^{i}} \frac{\left.\left.f\right|_{x^{i}=t} g\right|_{x^{i}=s}}{(s-t)^{1-\alpha_{i}}} d s d t, & \delta=+1, \\ f g, & \delta=0, \\ \frac{1}{\Gamma\left(\beta_{i}\right)} \int_{a^{i}}^{x^{i}} \int_{x^{i}}^{b^{i}} \frac{\left.\left.f\right|_{x^{i}=s} g\right|_{x^{i}=t}}{(s-t)^{1-\beta_{i}}} d s d t, & \delta=-1 .\end{cases}
$$

Оператор $J_{(i)}^{\delta}$ связан с оператором $B_{i}^{\delta}$ следующим тождеством:

$$
D_{i}\left(J_{(i)}^{\delta}\{f, g\}\right)=B_{i}^{\delta} f \cdot g-f \cdot\left(B_{i}^{\delta}\right)^{*} g .
$$

Справедливость данного равенства легко проверяется непосредственным дифференцированием правой части равенства (2.17). 
2.3. Группы точечных преобразований. Пусть $G$ - однопараметрическая группа точечных преобразований

$$
\begin{aligned}
x^{i} & =\varphi^{i}(x, u, \varepsilon), & \left.\varphi^{i}\right|_{\varepsilon=0} & =x^{i} \quad(i=1,2, \ldots, n), \\
u^{\prime} & =\psi(x, u, \varepsilon), & \left.\psi\right|_{\varepsilon=0} & =u
\end{aligned}
$$

и

$$
X=\xi^{i} \frac{\partial}{\partial x^{i}}+\eta \frac{\partial}{\partial u}
$$

- соответствующий ей инфинитезимальный оператор (генератор) группы. Координаты оператора $X$ суть

$$
\xi^{i}=\left.\frac{\partial \varphi^{i}}{\partial \varepsilon}\right|_{\varepsilon=0}(i=1,2, \ldots, n), \quad \eta=\left.\frac{\partial \psi}{\partial \varepsilon}\right|_{\varepsilon=0} .
$$

В (2.20) и далее используется правило суммирования по повторяющимся индексам.

В классическом групповом анализе определяются первое, второе и последующие продолжения группы $G$ соответственно на дифференциальные переменные $u_{(1)}, u_{(2)}$ и т. д. Инфинитезимальный оператор группы $G_{(k)}$, продолженной на все дифференциальные переменные до $k$-го порядка включительно (так называемый продолженный оператор $k$-го порядка), имеет вид [7], [8]

$$
X_{(k)}=\xi^{i} \frac{\partial}{\partial x^{i}}+\eta \frac{\partial}{\partial u}+\sum_{s=1}^{k} \zeta_{i_{1} i_{2} \ldots i_{s}} \frac{\partial}{\partial u_{i_{1} i_{2} \ldots i_{s}}} .
$$

Координаты $\zeta_{i_{1} i_{2} \ldots i_{s}}$ продолженного оператора определяются формулами продолжения, которые могут быть записаны в виде [8]

$$
\zeta_{i_{1} i_{2} \ldots i_{s}}=D_{i_{1}} D_{i_{2}} \ldots D_{i_{s}}(W)+\xi^{j} u_{j i_{1} i_{2} \ldots i_{s}}, \quad W=\eta-\xi^{j} u_{j}
$$

Продолжение группы $G$ на интегралы и производные дробного порядка обсуждается в работах [9]-[11]. Там же построены формулы продолжения для соответствующих координат продолженного инфинитезимального оператора группы. На основе этих результатов можно показать, что инфинитезимальный оператор группы $G_{[k]}$, продолженной на все линейные дробно-интегро-дифференциальные переменные (2.11) до $k$-го порядка включительно, имеет вид

$$
X_{[k]}=\xi^{i} \frac{\partial}{\partial x^{i}}+\eta \frac{\partial}{\partial u}+\sum_{s=1}^{k} \zeta_{i_{1} i_{2} \ldots i_{s}}^{\delta_{1} \delta_{2} \ldots \delta_{s}} \frac{\partial}{\partial u_{i_{1} i_{2} \ldots i_{s}}^{\delta_{1} \delta_{2} \ldots \delta_{s}}}
$$

где

$$
\zeta_{i_{1} i_{2} \ldots i_{s}}^{\delta_{1} \delta_{2} \ldots \delta_{s}}=B_{i_{s}}^{\delta_{s}} \ldots B_{i_{2}}^{\delta_{2}} B_{i_{1}}^{\delta_{1}}(W)+\xi^{j} D_{j}\left(u_{i_{1} i_{2} \ldots i_{s}}^{\delta_{1} \delta_{2} \ldots \delta_{s}}\right)
$$

В (2.22) и далее предполагается суммирование по повторяющимся индексам не только пространственных переменных $i_{r}$, но и по индексам операторов $\delta_{r}(r=1,2, \ldots, s)$. 


\section{3. ФУНДАМЕНТАЛЬНОЕ ОПЕРАТОРНОЕ ТОЖДЕСТВО}

Рассмотрим в области $\Omega(2.9)$ функционал $\Phi[u]$, заданный в виде вариационного интеграла с линейно-дробно-интегро-дифференциальной функцией $\mathcal{L} k$-го порядка в качестве подынтегральной функции:

$$
\Phi[u]=\int_{\Omega} \mathcal{L}\left(x, u, u_{[1]}, u_{[2]}, \ldots, u_{[k]}\right) d x, \quad \mathcal{L} \in \mathcal{F} .
$$

В механике функционал (3.1) традиционно называется интегралом действия, а функция $\mathcal{L}$ - функцией Лангранжа, или лагранжианом.

Рассмотрим задачу нахождения экстремума функционала (3.1) в предположении, что функция $u(x)$, на которой достигается экстремум, удовлетворяет следующим условиям на границе области $\Omega$ :

$$
\begin{aligned}
\left.u\left(x^{1}, \ldots, x^{n}\right)\right|_{x^{r}=a^{r}} & =\mu_{r}^{a}\left(x^{1}, \ldots, x^{r-1}, x^{r+1}, \ldots, x^{n}\right), \\
\left.u\left(x^{1}, \ldots, x^{n}\right)\right|_{x^{r}=b^{r}} & =\mu_{r}^{b}\left(x^{1}, \ldots, x^{r-1}, x^{r+1}, \ldots, x^{n}\right), \\
\left.B_{i_{s}}^{\delta_{s}} \ldots B_{i_{1}}^{\delta_{1}}(u)\right|_{x^{r}=a^{r}} & =\mu_{r i_{1} \ldots i_{s}}^{a \delta_{1} \ldots \delta_{s}}\left(x^{1}, \ldots, x^{r-1}, x^{r+1}, \ldots, x^{n}\right), \\
\left.B_{i_{s}}^{\delta_{s}} \ldots B_{i_{1}}^{\delta_{1}}(u)\right|_{x^{r}=b^{r}} & =\mu_{r i_{1} \ldots i_{s}}^{b \delta_{1} \ldots \delta_{s}}\left(x^{1}, \ldots, x^{r-1}, x^{r+1}, \ldots, x^{n}\right)
\end{aligned}
$$

$\left(i_{1}, \ldots, i_{s}=1,2, \ldots, n, \quad \delta_{1}, \ldots, \delta_{s}=-1,0,1, \quad s=1,2, \ldots, k-1, \quad r=1,2, \ldots, n\right)$, где $\mu_{r \ldots}^{a \ldots}, \mu_{r \ldots}^{b \ldots}-$ некоторые заданные функции. Отметим, что такая постановка граничных условий является естественной для уравнений с производными дробного порядка.

ЛЕмма 1. Для того чтобы функиионал (3.1), определенный на множестве функицй $и(x) \in A C^{k}(\Omega)$, удовлетворяющих условиям (3.2), достигал на данной функици и $(x)$ своего экстремального значения, необходимо, чтобы эта функция удовлетворяла уравнению Эйлера-Лагранжа

$$
\frac{\delta \mathcal{L}}{\delta u}=0
$$

в котором оператор вариационной производной $\delta / \delta и$ определяется равенством

$$
\frac{\delta}{\delta u}=\frac{\partial}{\partial u}+\sum_{s=1}^{k}\left(B_{i_{1}}^{\delta_{1}}\right)^{*}\left(B_{i_{2}}^{\delta_{2}}\right)^{*} \ldots\left(B_{i_{s}}^{\delta_{s}}\right)^{*} \frac{\partial}{\partial u_{i_{1} i_{2} \ldots i_{s}}^{\delta_{1} \delta_{2} \ldots \delta_{s}}} .
$$

ДокАЗАтЕЛЬство. В вариационном исчислении доказывается (см., например, книгу [25], с. 289), что необходимым условием экстремума функционала является обращение в нуль его вариации:

$$
\left.\delta \Phi[u(x)] \equiv\left(\frac{\partial}{\partial \varepsilon} \Phi[u+\varepsilon \delta u]\right)\right|_{\varepsilon=0}=0 .
$$

В силу линейности операторов (2.10) для функционала (3.1) находим

$$
\begin{aligned}
\delta \Phi[u(x)] & =\left.\left(\frac{\partial}{\partial \varepsilon} \int_{\Omega} \mathcal{L}\left(x, u+\varepsilon \delta u, u_{[1]}+\varepsilon \delta u_{[1]}, \ldots, u_{[k]}+\varepsilon \delta u_{[k]}\right) d x\right)\right|_{\varepsilon=0}= \\
& =\int_{\Omega}\left[\frac{\partial \mathcal{L}}{\partial u}+\sum_{s=1}^{k} \frac{\partial \mathcal{L}}{\partial u_{i_{1} i_{2} \ldots i_{s}}^{\delta_{1} \ldots \delta_{s}}} B_{i_{s}}^{\delta_{s}} \ldots B_{i_{1}}^{\delta_{1}}(\delta u)\right] d x,
\end{aligned}
$$


где $\delta u_{[s]}=\left\{B_{i_{s}}^{\delta_{s}} \ldots B_{i_{1}}^{\delta_{1}}(\delta u)\right\}(s=1,2, \ldots, k)$. Далее воспользуемся классической формулой интегрирования по частям целого порядка и известной [1], [2] формулой дробного интегрирования по частям

$$
\int_{a}^{b} \varphi(x)_{a} I_{x}^{\alpha} \psi(x) d x=\int_{a}^{b}{ }_{x} I_{b}^{\alpha} \varphi(x) \psi(x) d x .
$$

Имеем

$$
\begin{aligned}
& \delta \Phi[u(x)]=\sum_{s=1}^{k}\left\{\int_{\partial \Omega_{i_{1}}}\left[B_{i_{2}}^{\delta_{2}} \ldots B_{i_{s}}^{\delta_{s}}\left(\frac{\partial \mathcal{L}}{\partial u_{i_{1} i_{2} \ldots \delta_{s}}^{0 \delta_{2} \ldots \delta_{s}}}\right) \delta u\right]_{x^{i_{1}=a^{i_{1}}}}^{x^{i_{1}}=b^{i_{1}}} d x+\right. \\
& +\sum_{r=2}^{s-1} \int_{\partial \Omega_{i_{r}}}\left[B_{i_{r+1}}^{\delta_{r+1}} \ldots B_{i_{s}}^{\delta_{s}}\left(\frac{\partial \mathcal{L}}{\partial u_{i_{1} \ldots i_{r-1} i_{r} i_{r+1} \ldots i_{s}}^{\delta_{1} \ldots \delta_{r-1} 0 \delta_{r+1} \ldots \delta_{s}}}\right) \cdot B_{i_{r-1}}^{\delta_{r-1}} \ldots B_{i_{1}}^{\delta_{1}}(\delta u)\right]_{x^{i_{r}}=a^{i_{r}}}^{x^{i_{r}}=b^{i_{r}}} d x+ \\
& \left.+\int_{\partial \Omega_{i_{s}}}\left[\frac{\partial \mathcal{L}}{\partial u_{i_{1} \ldots i_{s-1} i_{s}}^{\delta_{1} \ldots \delta_{s-1} 0}} \cdot B_{i_{s-1}}^{\delta_{s-1}} \ldots B_{i_{1}}^{\delta_{1}}(\delta u)\right]_{x^{i_{s}=a^{i_{s}}}}^{x^{i_{s}}=b^{i_{s}}} d x\right\}+ \\
& +\int_{\Omega}\left[\frac{\partial \mathcal{L}}{\partial u}+\sum_{s=1}^{k}\left(B_{i_{1}}^{\delta_{1}}\right)^{*}\left(B_{i_{2}}^{\delta_{2}}\right)^{*} \ldots\left(B_{i_{s}}^{\delta_{s}}\right)^{*} \frac{\partial \mathcal{L}}{\partial u_{i_{1} i_{2} \ldots i_{s}}^{\delta_{1} \delta_{2} \ldots \delta_{s}}}\right] \delta u d x
\end{aligned}
$$

где $\delta \Omega_{r}=\Omega \backslash\left[a^{r}, b^{r}\right](r=1,2, \ldots, n)$. В силу условий $(3.2)$

$$
\begin{gathered}
\left.(\delta u)\right|_{x^{r}=a^{r}}=0,\left.\quad \delta u\right|_{x^{r}=b^{r}}=0 \\
\left.B_{i_{s}}^{\delta_{s}} \ldots B_{i_{1}}^{\delta_{1}}(\delta u)\right|_{x^{r}=a^{r}}=0,\left.\quad B_{i_{s}}^{\delta_{s}} \ldots B_{i_{1}}^{\delta_{1}}(\delta u)\right|_{x^{r}=b^{r}}=0 \\
\left(i_{1}, \ldots, i_{s}=1,2, \ldots, n, \quad \delta_{1}, \ldots, \delta_{s}=-1,0,1, \quad s=1,2, \ldots, k-1, \quad r=1,2, \ldots, n\right),
\end{gathered}
$$

поэтому все слагаемые в (3.5), кроме последнего интеграла, обращаются в нуль. Таким образом, решение рассматриваемой вариационной задачи приводит к равенству

$$
\delta \Phi[u(x)] \equiv \int_{\Omega}\left[\frac{\partial \mathcal{L}}{\partial u}+\sum_{s=1}^{k}\left(B_{i_{1}}^{\delta_{1}}\right)^{*}\left(B_{i_{2}}^{\delta_{2}}\right)^{*} \ldots\left(B_{i_{s}}^{\delta_{s}}\right)^{*} \frac{\partial \mathcal{L}}{\partial u_{i_{1} i_{2} \ldots i_{s}}^{\delta_{1} \delta_{2} \ldots \delta_{s}}}\right] \delta u d x=0
$$

откуда в силу основной леммы вариационного исчисления [25] и вытекает утверждение доказываемой леммы.

В групповом анализе доказывается (см., например, [7], [8]), что для дифференциальной функции $k$-го порядка вида (2.8) имеет место равенство

$$
X_{(k)}(F)+D_{i}\left(\xi^{i}\right) F=W \frac{\delta F}{\delta u}+D_{i}\left(\mathcal{N}_{(k)}^{i} F\right), \quad W=\eta-\xi^{j} u_{j}, \quad F \in \mathcal{A},
$$

известное как фундаментальное операторное тождество, или тождество Нётер. При этом операторы $\mathcal{N}_{(k)}^{i}$ (операторы Нётер [8]) определяются формулами

$$
\begin{aligned}
\mathcal{N}_{(k)}^{i} F= & \xi^{i} F+W\left(\frac{\partial F}{\partial u_{i}}+\sum_{s=1}^{k-1}(-1)^{s} D_{j_{1}} \ldots D_{j_{s}} \frac{\partial F}{\partial u_{i j_{1} \ldots j_{s}}}\right)+ \\
& +\sum_{r=1}^{k-1} D_{l_{1}} \ldots D_{l_{r}}(W)\left[\frac{\partial F}{\partial u_{i l_{1} \ldots l_{r}}}+\sum_{s=1}^{k-1-r}(-1)^{s} D_{j_{1}} \ldots D_{j_{s}} \frac{\partial F}{\partial u_{i l_{1} \ldots l_{r} j_{1} \ldots j_{s}}}\right] .
\end{aligned}
$$


Докажем, что для введенного класса функций вида (2.12) имеет место тождество, аналогичное (3.6).

Теорема 1. Справедливо равенство

$$
X_{[k]}(F)+D_{i}\left(\xi^{i}\right) F=W \frac{\delta F}{\delta u}+D_{i}\left(\mathcal{N}_{[k]}^{i} F\right), \quad W=\eta-\xi^{i} u_{i}, \quad F \in \mathcal{F}
$$

в котором операторы $X_{[k]}$ и $\delta / \delta и$ имеют соответственно вид (2.22) u (3.4), а операторьь $\mathcal{N}_{[k]}^{i}($ операторы Нётер дробного порядка) определяются равенствами

$$
\begin{aligned}
\mathcal{N}_{[k]}^{i} F & =\xi^{i} F+J_{(i)}^{\delta_{1}}\left\{W, \frac{\partial F}{\partial u_{i}^{\delta_{1}}}\right\}+\sum_{s=2}^{k}\left[J_{(i)}^{\delta_{1}}\left\{W,\left(B_{j_{2}}^{\delta_{2}}\right)^{*} \ldots\left(B_{j_{s}}^{\delta_{s}}\right)^{*} \frac{\partial F}{\partial u_{i j_{2} \ldots j_{s}}^{\delta_{1} \delta_{2} \ldots \delta_{s}}}\right\}+\right. \\
& \left.+\sum_{r=2}^{s} J_{(i)}^{\delta_{r}}\left\{B_{j_{r-1}}^{\delta_{r-1}} \ldots B_{j_{1}}^{\delta_{1}}(W),\left(B_{j_{r+1}}^{\delta_{r+1}}\right)^{*} \ldots\left(B_{j_{s}}^{\delta_{s}}\right)^{*} \frac{\partial F}{\partial u_{j_{1} \ldots j_{r-1} i j_{r+1} \ldots j_{s}}^{\delta_{1} \ldots \delta_{r-1} \delta_{\delta_{2}} \ldots \delta_{s}}}\right\}\right] .
\end{aligned}
$$

ДоказАтельство. С учетом определений (2.22) и (3.4) операторов $X_{[k]}$ и $\delta / \delta u$ имеем

$$
\begin{aligned}
D_{i}\left(\xi^{i}\right) F+X_{[k]}(F)-W \frac{\delta F}{\delta u}= \\
=D_{i}\left(\xi^{i}\right) F+\xi^{i} \frac{\partial F}{\partial x^{i}}+\eta \frac{\partial F}{\partial u}+\sum_{s=1}^{k}\left[B_{j_{s}}^{\delta_{s}} \ldots B_{j_{1}}^{\delta_{1}}(W)+\xi^{i} D_{i}\left(u_{j_{1} \ldots j_{s}}^{\delta_{1} \ldots \delta_{s}}\right)\right] \frac{\partial F}{\partial u_{j_{1} \ldots j_{s}}^{\delta_{1} \ldots \delta_{s}}}- \\
\quad-\left(\eta-\xi^{i} u_{i}\right) \frac{\partial F}{\partial u}-W \sum_{s=1}^{k}\left(B_{j_{1}}^{\delta_{1}}\right)^{*} \ldots\left(B_{j_{s}}^{\delta_{s}}\right)^{*} \frac{\partial F}{\partial u_{j_{1} \ldots j_{s}}^{\delta_{1} \ldots \delta_{s}}}= \\
=D_{i}\left(\xi^{i}\right) F+\xi^{i}\left(\frac{\partial F}{\partial x^{i}}+u_{i} \frac{\partial F}{\partial u}+\sum_{s=1}^{k} D_{i}\left(u_{j_{1} \ldots j_{s}}^{\delta_{1} \ldots \delta_{s}}\right) \frac{\partial F}{\partial u_{j_{1} \ldots j_{s}}^{\delta_{1} \ldots \delta_{s}}}\right)+ \\
\quad+\sum_{s=1}^{k}\left[B_{j_{s}}^{\delta_{s}} \ldots B_{j_{1}}^{\delta_{1}}(W) \cdot \frac{\partial F}{\partial u_{j_{1} \ldots j_{s}}^{\delta_{1} \ldots \delta_{s}}}-W \cdot\left(B_{j_{1}}^{\delta_{1}}\right)^{*} \ldots\left(B_{j_{s}}^{\delta_{s}}\right)^{*} \frac{\partial F}{\partial u_{j_{1} \ldots j_{s}}^{\delta_{1} \ldots \delta_{s}}}\right]= \\
=D_{i}\left(\xi^{i} F\right)+\sum_{s=1}^{k}\left[B_{j_{s}}^{\delta_{s}} \ldots B_{j_{1}}^{\delta_{1}}(W) \cdot \frac{\partial F}{\partial u_{j_{1} \ldots j_{s}}^{\delta_{1} \ldots \delta_{s}}}-W \cdot\left(B_{j_{1}}^{\delta_{1}}\right)^{*} \ldots\left(B_{j_{s}}^{\delta_{s}}\right)^{*} \frac{\partial F}{\partial u_{j_{1} \ldots j_{s}}^{\delta_{1} \ldots \delta_{s}}}\right] .
\end{aligned}
$$

Здесь при получении последнего равенства использовано определение оператора полного дифференцирования (2.14) по переменной $x^{i}$.

Рассмотрим выражение в правой части равенства (3.10) под знаком суммы. Для $s=1$ в силу (2.18) имеем

$$
B_{j_{1}}^{\delta_{1}}(W) \cdot \frac{\partial F}{\partial u_{j_{1}}^{\delta_{1}}}-W \cdot\left(B_{j_{1}}^{\delta_{1}}\right)^{*} \frac{\partial F}{\partial u_{j_{1}}^{\delta_{1}}}=D_{j_{1}}\left(J_{\left(j_{1}\right)}^{\delta_{1}}\left\{W, \frac{\partial F}{\partial u_{j_{1}}^{\delta_{1}}}\right\}\right)
$$


Далее для сокращения выкладок введем обозначения

$$
\begin{gathered}
f_{\delta_{1} \ldots \delta_{s}}^{j_{1} \ldots j_{s}} \equiv \frac{\partial F}{\partial u_{j_{1} \ldots j_{s}}^{\delta_{1} \ldots \delta_{s}}}, \quad f_{\delta_{1} \ldots \delta_{r}}^{j_{1} \ldots j_{r}} \equiv\left(B_{j_{r+1}}^{\delta_{r+1}}\right)^{*} \ldots\left(B_{j_{s}}^{\delta_{s}}\right)^{*} f_{\delta_{1} \ldots \delta_{s}}^{j_{1} \ldots j_{s}} \\
W_{j_{1} \ldots j_{r}}^{\delta_{1} \ldots \delta_{r}} \equiv B_{j_{r}}^{\delta_{r}} \ldots B_{j_{1}}^{\delta_{1}}(W) \\
(r=1,2, \ldots, s-1, \quad s=2,3, \ldots, k) .
\end{gathered}
$$

Тогда для $s>1$ в силу (2.18) получаем следующую цепочку равенств:

$$
\begin{aligned}
& B_{j_{s}}^{\delta_{s}} \ldots B_{j_{1}}^{\delta_{1}}(W) \cdot \frac{\partial F}{\partial u_{j_{1} \ldots j_{s}}^{\delta_{1} \ldots \delta_{s}}} \equiv B_{j_{s}}^{\delta_{s}}\left(W_{j_{1} \ldots j_{s-1}}^{\delta_{1} \ldots \delta_{s-1}}\right) \cdot f_{\delta_{1} \ldots \delta_{s}}^{j_{1} \ldots j_{s}}= \\
& =D_{j_{s}}\left(J_{\left(j_{s}\right)}^{\delta_{s}}\left\{W_{j_{1} \ldots j_{s-1}}^{\delta_{1} \ldots \delta_{s-1}}, f_{\delta_{1} \ldots \delta_{s}}^{j_{1} \ldots j_{s}}\right\}\right)+W_{j_{1} \ldots j_{s-1}}^{\delta_{1} \ldots \delta_{s-1}} \cdot\left(B_{j_{s}}^{\delta_{s}}\right)^{*} f_{\delta_{1} \ldots \delta_{s}}^{j_{1} \ldots j_{s}}= \\
& =D_{j_{s}}\left(J_{\left(j_{s}\right)}^{\delta_{s}}\left\{W_{j_{1} \ldots j_{s-1}}^{\delta_{1} \ldots \delta_{s-1}}, f_{\delta_{1} \ldots \delta_{s}}^{j_{1} \ldots j_{s}}\right\}\right)+B_{j_{s-1}}^{\delta_{s-1}}\left(W_{j_{1} \ldots j_{s-2}}^{\delta_{1} \ldots \delta_{s-2}}\right) \cdot f_{\delta_{1} \ldots \delta_{s-1}}^{j_{1} \ldots j_{s-1}}= \\
& =D_{j_{s}}\left(J_{\left(j_{s}\right)}^{\delta_{s}}\left\{W_{j_{1} \ldots j_{s-1}}^{\delta_{1} \ldots \delta_{s-1}}, f_{\delta_{1} \ldots \delta_{s}}^{j_{1} \ldots j_{s}}\right\}\right)+D_{j_{s-1}}\left(J_{\left(j_{s-1}\right)}^{\delta_{s-1}}\left\{W_{j_{1} \ldots j_{s-2}}^{\delta_{1} \ldots \delta_{s-2}}, f_{\delta_{1} \ldots \delta_{s-1}}^{j_{1} \ldots j_{s-1}}\right\}\right)+ \\
& +W_{j_{1} \ldots j_{s-2}}^{\delta_{1} \ldots \delta_{s-2}} \cdot f_{\delta_{1} \ldots \delta_{s-2}}^{j_{1} \ldots j_{s-2}}=\cdots= \\
& =D_{j_{s}}\left(J_{\left(j_{s}\right)}^{\delta_{s}}\left\{W_{j_{1} \ldots j_{s-1}}^{\delta_{1} \ldots \delta_{s-1}}, f_{\delta_{1} \ldots \delta_{s}}^{j_{1} \ldots j_{s}}\right\}\right)+\sum_{r=2}^{s-1} D_{j_{r}}\left(J_{\left(j_{r}\right)}^{\delta_{j_{r}}}\left\{W_{j_{1} \ldots j_{r-1}}^{\delta_{1} \ldots \delta_{r-1}}, f_{\delta_{1} \ldots \delta_{r}}^{j_{1} \ldots j_{r}}\right\}\right)+ \\
& +D_{j_{1}}\left(J_{\left(j_{1}\right)}^{\delta_{1}}\left\{W, f_{\delta_{1}}^{j_{1}}\right\}\right)+W \cdot\left(B_{j_{1}}^{\delta_{1}}\right)^{*} f_{\delta_{1}}^{j_{1}}= \\
& =D_{i}\left(J_{(i)}^{\delta_{1}}\left\{W, f_{\delta_{1}}^{i}\right\}+\sum_{r=2}^{s-1} J_{(i)}^{\delta_{r}}\left\{W_{j_{1} \ldots j_{r-1}}^{\delta_{1} \ldots \delta_{r-1}}, f_{\delta_{1} \ldots \delta_{r-1} \delta_{r}}^{j_{1} \ldots j_{r-1}, i}\right\}+\right. \\
& \left.+J_{(i)}^{\delta_{s}}\left\{W_{j_{1} \ldots j_{s-1}}^{\delta_{1} \ldots \delta_{s-1}}, f_{\delta_{1} \ldots \delta_{s-1} \delta_{s}}^{j_{1} \ldots j_{s-1} i}\right\}\right)+W \cdot\left(B_{j_{1}}^{\delta_{1}}\right)^{*} \ldots\left(B_{j_{s}}^{\delta_{s}}\right)^{*} f_{\delta_{1} \ldots \delta_{s}}^{j_{1} \ldots j_{s}} .
\end{aligned}
$$

Подставляя полученные результаты в (3.10), с учетом обратной замены в силу (3.11) приходим к равенству

$$
\begin{aligned}
& D_{i}\left(\xi^{i}\right) F+X_{[k]}(F)-W \frac{\delta F}{\delta u}= \\
& =D_{i}\left(\xi^{i} F\right)+D_{i}\left(J_{(i)}^{\delta_{1}}\left\{W, \frac{\partial F}{\partial u_{i}^{\delta_{1}}}\right\}\right)+D_{i}\left(\sum _ { s = 2 } ^ { k } \left[J_{(i)}^{\delta_{1}}\left\{W,\left(B_{j_{2}}^{\delta_{2}}\right)^{*} \ldots\left(B_{j_{s}}^{\delta_{s}}\right)^{*} \frac{\partial F}{\partial u_{i j_{2} \ldots j_{s}}^{\delta_{1} \delta_{2} \ldots \delta_{s}}}\right\}+\right.\right. \\
& \quad+\sum_{r=2}^{s} J_{(i)}^{\delta_{r}}\left\{B_{j_{r-1}}^{\delta_{r-1}} \ldots B_{j_{1}}^{\delta_{1}}(W),\left(B_{j_{r+1}}^{\delta_{r+1}}\right)^{*} \ldots\left(B_{j_{s}}^{\delta_{s}}\right)^{*} \frac{\partial F}{\left.\left.\left.\partial u_{j_{1} \ldots j_{r-1} i j_{r+1} \ldots j_{s}}^{\delta_{1} \ldots \delta_{r-1} \delta_{r} \delta_{r+1} \ldots \delta_{s}}\right\}\right]\right)=}\right. \\
& =D_{i}\left(\mathcal{N}_{[k]}^{i} F\right),
\end{aligned}
$$

где $\mathcal{N}_{[k]}^{i} F$ имеет вид (3.9). Тождество (3.8) доказано.

В заключение данного раздела выпишем операторы Нётер (3.9) для часто встречающихся на практике видов функций $F$, зависящих от одной независимой переменной $x$ и от производных типа Римана-Лиувилля и Капуто дробного порядка $\alpha>0$. Положим $m=[\alpha]+1$. В силу определений (2.3)-(2.6) операторы дробного дифференцирования Римана-Лиувилля и Капуто представляются в виде произведения $m+1$ операторов вида (2.10), а именно $m$ операторов дифференцирования и одного 
оператора интегрирования дробного порядка $m-\alpha$. Поэтому все приведенные ниже функции $F$ являются линейно-дробно-интегро-дифференциальными функциями порядка $k=m+1$. Имеем

$$
\begin{aligned}
& F=F\left(x, u,{ }_{a} D_{x}^{\alpha} u\right): \\
& \mathcal{N}_{[m+1]}^{x} F=\xi^{x} F+\sum_{r=1}^{m-1}(-1)^{r-1}{ }_{a} D_{x}^{\alpha-r}(W) \cdot D_{x}^{r-1} \frac{\partial F}{\partial\left({ }_{a} D_{x}^{\alpha} u\right)}+ \\
& +(-1)^{m-1}{ }_{a} I_{x}^{m-\alpha}(W) \cdot D_{x}^{m-1}\left(\frac{\partial F}{\partial\left({ }_{a} D_{x}^{\alpha} u\right)}\right)+(-1)^{m} J_{(x)}^{+1}\left\{W, D_{x}^{m} \frac{\partial F}{\partial\left({ }_{a} D_{x}^{\alpha} u\right)}\right\}, \\
& F=F\left(x, u,{ }_{x} D_{b}^{\alpha} u\right): \\
& \mathcal{N}_{[m+1]}^{x} F=\xi^{x} F-\sum_{r=1}^{m-1}{ }_{x} D_{b}^{\alpha-r}(W) \cdot D_{x}^{r-1} \frac{\partial F}{\partial\left({ }_{x} D_{b}^{\alpha} u\right)}- \\
& -{ }_{x} I_{b}^{m-\alpha}(W) \cdot D_{x}^{m-1}\left(\frac{\partial F}{\partial\left({ }_{x} D_{b}^{\alpha} u\right)}\right)+J_{(x)}^{-1}\left\{W, D_{x}^{m} \frac{\partial F}{\partial\left(_{x} D_{b}^{\alpha} u\right)}\right\} \\
& F=F\left(x, u,{ }_{a}^{\mathrm{C}} D_{x}^{\alpha} u\right): \\
& \mathcal{N}_{[m+1]}^{x} F=\xi^{x} F+\sum_{r=1}^{m-1} D_{x}^{r-1} W \cdot{ }_{x} D_{b}^{\alpha-r} \frac{\partial F}{\partial\left({ }_{a}^{\mathrm{C}} D_{x}^{\alpha} u\right)}+ \\
& +D_{x}^{m-1}(W) \cdot{ }_{x} I_{b}^{m-\alpha} \frac{\partial F}{\partial\left({ }_{a}^{\mathrm{C}} D_{x}^{\alpha} u\right)}+J_{(x)}^{+1}\left\{D_{x}^{m}(W), \frac{\partial F}{\partial\left({ }_{a}^{\mathrm{C}} D_{x}^{\alpha} u\right)}\right\}, \\
& F=F\left(x, u,{ }_{x}^{\mathrm{C}} D_{b}^{\alpha} u\right): \\
& \mathcal{N}_{[m+1]}^{x} F=\xi^{x} F+\sum_{r=1}^{m-1}(-1)^{r-1} D_{x}^{r-1}(W) \cdot{ }_{a} D_{x}^{\alpha-r} \frac{\partial F}{\partial\left({ }_{x}^{\mathrm{C}} D_{b}^{\alpha} u\right)}+ \\
& +(-1)^{m-1} D_{x}^{m-1}(W) \cdot{ }_{a} I_{x}^{m-\alpha} \frac{\partial F}{\partial\left({ }_{x}^{\mathrm{C}} D_{b}^{\alpha} u\right)}+(-1)^{m} J_{(x)}^{-1}\left\{D_{x}^{m}(W), \frac{\partial F}{\partial\left({ }_{x}^{\mathrm{C}} D_{b}^{\alpha} u\right)}\right\} \text {. }
\end{aligned}
$$

В данном случае порядок дробного интегрирования в операторах $J_{(x)}^{ \pm 1}$ равен $m-\alpha$.

Заметим, что для частного случая $\alpha \in(0,1)$ равенство $(3.12)$ совпадает с аналогичной формулой, полученной в работе [15].

\section{4. ЗАКОНЫ СОХРАНЕНИЯ ДЛЯ УРАВНЕНИЙ С ИНТЕГРАЛАМИ И ПРОИЗВОДНЫМИ ДРОБНОГО ПОРЯДКА}

В настоящей работе для интегро-дифференциальных уравнений дробного порядка будет использоваться классическое определение закона сохранения.

ОПРЕДЕЛЕНИЕ 8. Равенство

$$
D_{i}\left(C^{i}\right)=0
$$

называется законом сохранения для интегро-дифференциального уравнения дробного порядка, если оно выполняется тождественно на любом решении этого уравнения. Векторное поле $C=\left(C^{1}, \ldots, C^{n}\right)$, удовлетворяющее $(4.1)$, называется сохраняющимся вектором. 
Рассмотрим проблему построения законов сохранения для интегро-дифференциальных уравнений дробного порядка, являющихся уравнениями Эйлера-Лагранжа (3.3) для функционала вида $(3.1)$ с лагранжианом $\mathcal{L} \in \mathcal{F}$. Отметим, что уравнение Эйлера-Лагранжа с таким лагранжианом в общем случае не сводится к равенству нулю некоторой линейно-дробно-интегро-дифференциальной функции.

Теорема 1 позволяет доказать аналог теоремы Нётер для функционала (3.1). Как показано в работе [13] (см. также [7], [8]), для упрощения доказательства теоремы Нётер вместо рассмотрения инвариантности функционала (3.1) относительно группы $G$ точечных преобразований (2.19) достаточно рассмотреть инвариантность элементарного действия $\mathcal{L} d x$. Действие этой группы на элемент объема $d x$ определяется классическим образом [8], [13]:

$$
d x^{\prime}=\operatorname{det}\left(D_{i} x^{\prime k}\right) d x,\left.\quad \frac{\partial\left(d x^{\prime}\right)}{\partial \varepsilon}\right|_{\varepsilon=0}=D_{i}\left(\xi^{i}\right) d x .
$$

Тогда продолжение инфинитезимального оператора (2.20) группы точечных преобразований (2.19) на линейные дробно-интегро-дифференциальные переменные до $k$-го порядка включительно и на дополнительную независимую переменную $d x$ имеет вид

$$
\widetilde{X}_{[k]}=X_{[k]}+D_{i}\left(\xi^{i}\right) d x \frac{\partial}{\partial(d x)},
$$

где $X_{[k]}$ определяется формулой (2.22).

Для инвариантности элементарного действия относительно группы $G$ необходимо и достаточно выполнения равенства $\widetilde{X}_{[k]}(\mathcal{L} d x)=0$, или

$$
X_{[k]}(\mathcal{L})+D_{i}\left(\xi^{i}\right) \mathcal{L}=0 .
$$

С использованием доказанного тождества (3.8) это условие переписывается в виде

$$
W \frac{\delta \mathcal{L}}{\delta u}+D_{i}\left(\mathcal{N}_{[k]}^{i} \mathcal{L}\right)=0
$$

где операторы $\mathcal{N}_{[k]}^{i}$ определяются по формуле (3.9). В силу справедливости уравнения Эйлера-Лагранжа (3.3) первое слагаемое данного равенства обращается в нуль, и это равенство превращается в закон сохранения (4.1). Тем самым доказан следующий дробно-интегро-дифференциальный аналог теоремы Нётер.

Теорема 2. Пусть $\mathcal{L} \in \mathcal{F}$ и элементарное действие $\mathcal{L} d x$ инвариантно относительно группы точечных преобразований с оператором (4.2). Тогда функиии

$$
C^{i}=\mathcal{N}_{[k]}^{i} \mathcal{L} \quad(i=1,2, \ldots, n)
$$

являются компонентами сохраняющегося вектора для уравнения Эйлера-Лагранжа (3.3). В соотношении (4.4) операторы $\mathcal{N}_{[k]}^{i}$ определяются равенством (3.9).

Таким образом, знание явного вида (3.9) операторов Нётер дает эффективный способ построения законов сохранения для уравнений с дробно-дифференциальными операторами, являющимися уравнениями Эйлера-Лагранжа с линейно-дробно-интегро-дифференциальным лагранжианом, симметрии которых удовлетворяют условию инвариантности (4.3). 
ЗАмЕчАниЕ 2. Как и в случае дифференциальных уравнений целого порядка, условие инвариантности (4.3) может быть заменено условием дивергентности

$$
X_{[k]} \mathcal{L}+D_{i}\left(\xi^{i}\right) \mathcal{L}=D_{i}\left(H^{i}\right)
$$

с некоторыми функциями $H^{i}(i=1,2, \ldots, n)$. Тогда компоненты сохраняющегося вектора имеют вид

$$
C^{i}=\mathcal{N}_{[k]}^{i} \mathcal{L}-H^{i}
$$

Так же, как и для дифференциальных уравнений целого порядка, для дробноинтегро-дифференциальных уравнений теорема 2 дает лишь достаточное условие существования законов сохранения. Аналогично тому, как это сделано в работах [7], [8], [13], данную теорему можно усилить.

Теорема 3. Для уравнения Эйлера-Лагранжа (3.3) слагранжианом $\mathcal{L} \in \mathcal{F}$, допускающего группу $G$ точечных преобразований (2.19) с оператором (2.20), необходимым и достаточным условием существования закона сохранения вида (4.1), компоненты сохраняющегося вектора которого определяются в (4.4), является инвариантность функционала (3.1) относительно группь $G$ на многообразии, заданном уравнением (3.3), т.е. условие

$$
\left.\left(X_{[k]} \mathcal{L}+D_{i}\left(\xi^{i}\right) \mathcal{L}\right)\right|_{(3.3)}=0 .
$$

ДокАзАтельство. Для существования сохраняющегося вектора с компонентами (4.4) в силу тождества (3.6) равенство (4.3) с необходимостью должно быть выполнено на решениях уравнения Эйлера-Лагранжа (3.3). Достаточность условия непосредственно следует из теоремы 2.

\section{5. ПРИМЕРЫ ПОСТРОЕНИЯ ЗАКОНОВ СОХРАНЕНИЯ ДЛЯ УРАВНЕНИЙ С ПРОИЗВОДНЫМИ ДРОБНОГО ПОРЯДКА}

5.1. Дробный осциллятор. Рассмотрим уравнение

$$
{ }_{x} D_{1}^{\alpha}\left({ }_{0}^{\mathrm{C}} D_{x}^{\alpha} u\right)-\omega^{2} u=0, \quad x \in(0,1), \quad \alpha \in(0,1),
$$

которое является уравнением Эйлера-Лагранжа (3.3) с лагранжианом

$$
\mathcal{L}=\frac{1}{2}\left[\left({ }_{0}^{\mathrm{C}} D_{x}^{\alpha} u\right)^{2}-\omega^{2} u^{2}\right]
$$

Уравнение (5.1) известно в дробной динамике как уравнение дробного осциллятоpa [26], [27]. Один из законов сохранения этого уравнения, соответствующий оператору переноса $X=\partial / \partial x$, был найден в работе [15]. Построим этот же закон сохранения с использованием предлагаемого в настоящей работе подхода.

Используя (2.21), находим, что продолжение указанного оператора переноса на производную Капуто ${ }_{0}^{\mathrm{C}} D_{x}^{\alpha} u$ имеет вид

$$
X=\frac{\partial}{\partial x}+\left[-{ }_{0}^{\mathrm{C}} D_{x}^{\alpha}\left(u_{x}\right)+D_{x}\left({ }_{0}^{\mathrm{C}} D_{x}^{\alpha} u\right)\right] \frac{\partial}{\partial\left({ }_{0}^{\mathrm{C}} D_{x}^{\alpha} u\right)} .
$$


В силу известной связи между дробными производными типа Римана-Лиувилля и Капуто (см., например, монографию [2]) справедливо равенство

$$
{ }_{0}^{\mathrm{C}} D_{x}^{\alpha}\left(u_{x}\right)=D_{x}\left({ }_{0}^{\mathrm{C}} D_{x}^{\alpha} u\right)+\frac{1}{\Gamma(1-\alpha)} \frac{u_{x}(0)}{x^{\alpha}},
$$

с учетом которого получаем, что при выполнении условия $u_{x}(0)=0$ оператор переноса по $x$ имеет нулевое продолжение на дробную производную Капуто. В этом случае условие (4.3) инвариантности элементарного действия выполнено тождественно. Поэтому оператор $X=\partial / \partial x$ является условной вариационной симметрией уравнения (5.1) и может быть использован для построения закона сохранения этого уравнения.

Поскольку уравнение (5.1) представляет собой обыкновенное дифференциальное уравнение дробного порядка, закон сохранения является для него первым интегралом. Используя соотношения (4.4) и (3.14), для лагранжиана (5.2) получаем

$$
C^{x}=\mathcal{N}^{x} \mathcal{L}=\xi^{x} \mathcal{L}+W_{x} I_{1}^{1-\alpha}\left(\frac{\partial \mathcal{L}}{\partial\left({ }_{0}^{\mathrm{C}} D_{x}^{\alpha} u\right)}\right)+J_{(x)}^{+1}\left\{D_{x}(W), \frac{\partial \mathcal{L}}{\left.\partial{ }_{0}^{\mathrm{C}} D_{x}^{\alpha} u\right)}\right\} .
$$

Здесь порядок дробного интегрирования в операторе $J_{(x)}^{+1}$ равен $1-\alpha$. Для оператора переноса $X=\partial / \partial x$ имеем $\xi^{x}=1, W=-u_{x}$, поэтому

$$
C^{x}=\frac{1}{2}\left[\left({ }_{0}^{\mathrm{C}} D_{x}^{\alpha} u\right)^{2}-\omega^{2} u^{2}\right]-u_{x} I_{1}^{1-\alpha}\left({ }_{0}^{\mathrm{C}} D_{x}^{\alpha} u\right)+\frac{1}{\Gamma(1-\alpha)} \int_{0}^{x} \int_{x}^{1} \frac{u_{t t}{ }_{0}^{\mathrm{C}} D_{s}^{\alpha} u}{(s-t)^{\alpha}} d s d t .
$$

Последний интеграл можно преобразовать:

$$
\begin{aligned}
\frac{1}{\Gamma(1-\alpha)} & \int_{0}^{x} \int_{x}^{1} \frac{u_{t t}{ }_{0}^{\mathrm{C}} D_{s}^{\alpha} u}{(s-t)^{\alpha}} d s d t= \\
& =\frac{1}{\Gamma(1-\alpha)} \int_{0}^{x} u_{t t}\left[\int_{t}^{1} \frac{{ }_{0}^{\mathrm{C}} D_{s}^{\alpha} u}{(s-t)^{\alpha}} d s-\int_{t}^{x} \frac{{ }_{0}^{\mathrm{C}} D_{s}^{\alpha} u}{(s-t)^{\alpha}} d s\right] d t= \\
& =u_{x} I_{1}^{1-\alpha}\left({ }_{0}^{\mathrm{C}} D_{x}^{\alpha} u\right)+\int_{0}^{x}\left[u_{t} D_{t}^{\alpha}\left({ }_{0}^{\mathrm{C}} D_{t}^{\alpha} u\right)-{ }_{0}^{\mathrm{C}} D_{t}^{\alpha} u_{0} D_{t}^{\alpha}\left(u_{t}\right)\right] d t .
\end{aligned}
$$

В результате находим, что уравнение (5.1) допускает первый интеграл

$$
C^{x}=\frac{1}{2}\left[\left({ }_{0}^{\mathrm{C}} D_{x}^{\alpha} u\right)^{2}-\omega^{2} u^{2}\right]+\int_{0}^{x}\left[u_{t}{ }_{t} D_{1}^{\alpha}\left({ }_{0}^{\mathrm{C}} D_{t}^{\alpha} u\right)-{ }_{0}^{\mathrm{C}} D_{t}^{\alpha} u_{0} D_{t}^{\alpha}\left(u_{t}\right)\right] d t,
$$

который идентичен полученному в примере 18 из работы [15]. Интегрируя по частям второй интеграл, получаем

$$
\begin{aligned}
C^{x}= & -\frac{1}{2}\left[\left({ }_{0}^{\mathrm{C}} D_{x}^{\alpha} u\right)^{2}+\omega^{2} u^{2}\right]+\left.\left({ }_{0}^{\mathrm{C}} D_{x}^{\alpha} u\right)^{2}\right|_{x=0}+ \\
& +\int_{0}^{x}\left[u_{t}{ }_{t} D_{1}^{\alpha}\left({ }_{0}^{\mathrm{C}} D_{t}^{\alpha} u\right)+D_{t}\left({ }_{0}^{\mathrm{C}} D_{t}^{\alpha} u\right)_{0} D_{t}^{\alpha}\left(u_{t}\right)\right] d t .
\end{aligned}
$$

При $\alpha=1$ (предельный случай, соответствующий классическому осциллятору) второе и третье слагаемые в правой части равенства (5.4) обращаются в нуль, и $C^{x}$ представляет собой обычную сумму кинетической и потенциальной энергий осциллятора: $C^{x}=\left(u_{x}^{2}+\omega^{2} u^{2}\right) / 2$. Поэтому сохраняющаяся величина (5.4) является полной энергией дробного осциллятора, а соответствующий закон сохранения есть закон сохранения энергии. 
5.2. Стационарное уравнение переноса во фрактальных средах. Рассмотрим обыкновенное дифференциальное уравнение дробного порядка

$$
\mathcal{D}_{x}^{\alpha} u-\omega^{2} u=0, \quad x \in(-\infty, \infty), \quad \alpha \in(1,2),
$$

где

$$
\mathcal{D}_{x}^{\alpha} \equiv \frac{1}{2}\left(D_{x+}^{\alpha}+D_{x-}^{\alpha}\right)=\frac{1}{2}\left[D_{x}^{2}\left({ }_{-\infty} I_{x}^{2-\alpha}\right)+D_{x}^{2}\left({ }_{x} I_{+\infty}^{2-\alpha}\right)\right]
$$

есть симметричный оператор дробного дифференцирования, совпадающий (с точностью до постоянного множителя) с одномерным потенциалом Рисса дробного порядка [1]. В предельном случае $\alpha=2$ справедливо равенство $\mathcal{D}_{x}^{2}=D_{x}^{2}$.

Уравнения с оператором (5.6), действующим по пространственной переменной, используются, в частности, для моделирования пространственно-нелокального диффузионного переноса в турбулентном пограничном слое [28], а также процессов аномального переноса примеси в трещиновато-пористых горных породах [29] и в сильно неоднородных геологических формациях [30]. Уравнение (5.5) представляет собой стационарный случай одного из таких уравнений аномальной (т. е. не подчиняющейся закону Фика) диффузии. Оно возникает также при построении решения такого линейного уравнения аномальной диффузии с помощью интегрального преобразования Лапласа по временной переменной.

Решение уравнения (5.5) ищется в пространстве функций, интегрируемых на всей оси вместе со своими первыми производными. Тогда выполнены соотношения

$$
D_{x+}^{\alpha} u=I_{x+}^{1-\alpha / 2} D_{x}^{2} I_{x+}^{1-\alpha / 2} u, \quad D_{x-}^{\alpha} u=I_{x-}^{1-\alpha / 2} D_{x}^{2} I_{x-}^{1-\alpha / 2} u,
$$

и уравнение (5.5) является уравнением Эйлера-Лагранжа, соответствующим функционалу (3.1) с лагранжианом

$$
\mathcal{L}=\frac{1}{2}\left[-D_{x+}^{\alpha / 2} u D_{x-}^{\alpha / 2} u+\omega^{2} u^{2}\right]
$$

Уравнение (5.5) допускает группу переносов с оператором $X=\partial / \partial x$. Условие инвариантности (4.3) для этого оператора выполнено, поэтому теорема 2 применима.

Сохраняющийся вектор будет иметь в данном случае единственную компоненту, которую можно вычислить, используя соотношения (4.4), (3.12), (3.13):

$$
\begin{aligned}
C^{x}=\mathcal{N}^{x} \mathcal{L}= & \mathcal{L}-I_{x+}^{\beta}\left(u_{x}\right) \frac{\partial \mathcal{L}}{\partial\left(D_{x+}^{\alpha / 2} u\right)}+I_{x-}^{\beta}\left(u_{x}\right) \frac{\partial \mathcal{L}}{\partial\left(D_{x-}^{\alpha / 2} u\right)}+ \\
& +J_{(x)}^{+1}\left\{u_{x}, D_{x}\left(\frac{\partial \mathcal{L}}{\partial\left(D_{x+}^{\alpha / 2} u\right)}\right)\right\}-J_{(x)}^{-1}\left\{u_{x}, D_{x}\left(\frac{\partial \mathcal{L}}{\partial\left(D_{x-}^{\alpha / 2} u\right)}\right)\right\} .
\end{aligned}
$$

После подстановки лагранжиана (5.7), раскрытия операторов $J_{(x)}^{\delta}$ в соответствии с формулой (2.17) и выполнения преобразований, аналогичных тем, которые проводились в предыдущем примере, получаем

$$
\begin{aligned}
C^{x}= & -\frac{1}{2}\left\{D_{x+}^{\alpha / 2}(u) D_{x-}^{\alpha / 2}(u)+\omega^{2} u^{2}-\right. \\
& \left.-\int_{-\infty}^{x}\left[D_{t+}^{1+\alpha / 2}(u) D_{t-}^{\alpha / 2}(u)-D_{t-}^{1+\alpha / 2}(u) D_{t+}^{\alpha / 2}(u)+2 \omega^{2} u u_{t}\right] d t\right\} .
\end{aligned}
$$


В предельном случае $\alpha=2$ подынтегральное выражение в (5.8) в силу уравнения обращается в нуль и $C^{x}=\left(u_{x}^{2}-\omega^{2} u^{2}\right) / 2$, что является следствием закона сохранения массы. Аналогично, для стационарного уравнения аномальной диффузии (5.5) закон сохранения, соответствующий сохраняющейся величине (5.8), также является следствием закона сохранения массы.

5.3. Дробно-дифференциальное волновое уравнение. Рассмотрим аналог волнового уравнения с производными дробного порядка по пространственной переменной:

$$
u_{t t}=-{ }_{x}^{\mathrm{C}} D_{1}^{\alpha}\left({ }_{0} D_{x}^{\alpha} u\right), \quad x \in(0,1), \quad \alpha \in(0,1) .
$$

Данное уравнение описывает процесс распространения волн в средах с пространственной нелокальностью, характеризующейся показателем $\alpha$, и является частным случаем обобщенного дробно-дифференциального волнового уравнения, которое было недавно предложено в работе [31]. Предельный переход $\alpha \rightarrow 1-0$ переводит уравнение (5.9) в классическое волновое уравнение с производными целого порядка.

Уравнение (5.9) является уравнением Эйлера-Лагранжа (3.3) с лагранжианом

$$
\mathcal{L}=\frac{1}{2}\left[\left({ }_{0} D_{x}^{\alpha} u\right)^{2}-u_{t}^{2}\right]
$$

и обладает, в частности, следующими симметриями:

$$
X_{1}=\frac{\partial}{\partial t}, \quad X_{2}=u \frac{\partial}{\partial u}, \quad X_{3}=x^{\alpha-1} \frac{\partial}{\partial u}, \quad X_{4}=x^{\alpha} \frac{\partial}{\partial u} .
$$

Нетрудно проверить, что с данным лагранжианом операторы $X_{1}$ и $X_{3}$ удовлетворяют условию (4.3), а оператор $X_{4}$ удовлетворяет условию дивергентности (4.5) с $H^{x}=\Gamma(\alpha+1)_{0} I_{x}^{1-\alpha} u$. Оператор $X_{2}$ этим условиям не удовлетворяет.

Для лагранжиана (5.10) компоненты сохраняющегося вектора, определяющиеся по формуле (4.4) с учетом (3.9), имеют вид

$$
\begin{aligned}
C^{t} & =\mathcal{N}^{t} \mathcal{L}=\xi^{t} \mathcal{L}+W \frac{\partial \mathcal{L}}{\partial u_{t}} \\
C^{x} & =\mathcal{N}^{x} \mathcal{L}=\xi^{x} \mathcal{L}+{ }_{0} I_{x}^{1-\alpha}(W) \frac{\partial \mathcal{L}}{\partial\left({ }_{0} D_{x}^{\alpha} u\right)}-J_{(x)}^{+1}\left\{W, D_{x} \frac{\partial \mathcal{L}}{\partial\left({ }_{0} D_{x}^{\alpha} u\right)}\right\},
\end{aligned}
$$

где $W=\eta-\xi^{t} u_{t}-\xi^{x} u_{x}$, а $\xi^{t}, \xi^{x}, \eta$ - координаты соответствующего инфинитезимального оператора группы. Порядок дробного интегрирования в операторе $J_{(x)}^{+1}$ в данном случае равен $1-\alpha$.

Оператор $X_{1}$ дает $W=-u_{t}$ и порождает сохраняющийся вектор с компонентами

$$
C^{t}=\frac{u_{t}^{2}}{2}+\frac{\left({ }_{0} D_{x}^{\alpha}\right)^{2} u}{2}, \quad C^{x}=-\left({ }_{0} D_{x}^{\alpha} u\right)\left({ }_{0} I_{x}^{1-\alpha} u_{t}\right)+J_{(x)}^{+1}\left\{u_{t},{ }_{0} D_{x}^{\alpha+1} u\right\} .
$$

Соответствующий закон сохранения есть закон сохранения энергии.

Оператор $X_{3}$ дает $W=x^{\alpha-1}$ и порождает сохраняющийся вектор с компонентами

$$
C^{t}=-x^{\alpha-1} u_{t}, \quad C^{x}=\Gamma(\alpha)_{0} D_{x}^{\alpha} u-J_{(x)}^{+1}\left\{x^{\alpha-1},{ }_{0} D_{x}^{\alpha+1} u\right\} .
$$


Соответствующий закон сохранения является дробно-дифференциальным аналогом закона сохранения импульса, в который он переходит в предельном случае $\alpha=1$.

Оператор $X_{4}$ дает $W=x^{\alpha}$ и, с учетом замечания 2, порождает сохраняющийся вектор с компонентами

$$
C^{t}=-x^{\alpha} u_{t}, \quad C^{x}=\Gamma(1+\alpha)\left[x_{0} D_{x}^{\alpha} u-{ }_{0} I_{x}^{1-\alpha} u\right]-J_{(x)}^{+1}\left\{x^{\alpha},{ }_{0} D_{x}^{\alpha+1} u\right\} .
$$

Соответствующий закон сохранения является одним из следствий закона сохранения импульса.

Непосредственным дифференцированием нетрудно убедиться, что все найденные сохраняющиеся векторы удовлетворяют закону сохранения (4.1) в силу уравнения (5.9).

\section{6. ОБСУЖДЕНИЕ}

Результаты настоящей работы дают эффективный способ построения законов сохранения для интегро-дифференциальных уравнений дробного порядка, имеющих линейно-дробно-интегро-дифференциальный лагранжиан. Нахождение компонент сохраняющего вектора по известным симметриям сводится при этом к применению к лагранжиану уравнения операторов Нётер, полученных в работе в явном виде. При этом интересно отметить, что получающиеся в результате компоненты сохраняющегося вектора являются в общем случае нелокальными даже в функциональном пространстве $\mathcal{F}$, поскольку зависят от интегрального оператора $(2.17)$, который не может быть представлен через конечную комбинацию операторов интегрирования дробного порядка.

Несмотря на то что все результаты работы были получены для одиночного уравнения, они легко обобщаются на случай систем интегро-дифференциальных уравнений дробного порядка рассмотренного вида. Возможным продолжением работы является распространение полученных результатов на уравнения с дробными производными других типов, в том числе континуального порядка.

Однако одной из главных практических сложностей построения законов сохранения для большинства существующих в настоящее время физически обоснованных дробно-дифференциальных уравнений (таких, как уравнения субдиффузии, диффузионно-волновые уравнения, уравнения адвекции-диффузии дробного порядка и т. д.) является отсутствие у этих уравнений лагранжиана. Тем не менее, как показано в работе [8], для случая дифференциальных уравнений целого порядка данная трудность может быть преодолена путем перехода к так называемым слабым лагранжианам, которые всегда существуют для любого дифференциального уравнения. Доказанная в настоящей работе теорема 3 дает теоретическое обоснование распространения данного подхода и на интегро-дифференциальные уравнения дробного порядка.

Недавно в работах [32], [33] для дифференциальных уравнений целого порядка была предложена концепция нелинейной самосопряженности, в которой используется понятие формального лагранжиана как одной из разновидностей слабого лагранжиана. Развитый в этих работах подход позволяет использовать симметрии для построения законов сохранения в случае нелинейно-самосопряженных дифференциальных уравнений, не имеющих лагранжиана в классическом смысле. При 
этом применяются полученные ранее операторы Нётер (3.7). С использованием этого подхода были найдены новые семейства законов сохранения для большого количества различных дифференциальных уравнений, не имеющих классического лагранжиана. Результаты настоящей работы закладывают теоретическую основу для распространения концепции нелинейной самосопряженности на интегро-дифференциальные уравнения дробного порядка, что даст возможность построения законов сохранения для многих современных физически значимых моделей, которые можно представить такими уравнениями.

Благодарности. Автор выражает благодарность рецензентам за ряд ценных замечаний, позволивших улучшить эту работу. Работа выполнена при финансовой поддержке гранта Правительства РФ в рамках постановления № 220 (договор № 11.G34.31.0042).

\section{Список литературы}

[1] С. Г. Самко, А. А. Килбас, О. И. Маричев, Интеграль и производные дробного порядка и некоторые их приложения, Наука и техника, Минск, 1987.

[2] A. A. Kilbas, H. M. Srivastava, J. J. Trujillo, Theory and Applications of Fractional Differential Equations, North-Holland Mathematics Studies, 204, Elsevier, Amsterdam, 2006.

[3] R. Metzler, J. Klafter, Phys. Rep., 339:1 (2000), 1-77.

[4] R. Klages, G. Radons, I. M. Sokolov (eds.), Anomalous Transport: Foundations and Applications, Wiley-VCH, Berlin, 2008.

[5] J. Klafter, S. C. Lim, R. Metzler (eds.), Fractional Dynamics: Recent Advances, World Sci., Singapore, 2011.

[6] V.V. Uchaikin, Fractional Derivatives for Physicists and Engineers, Springer, Singapore, 2013.

[7] Л. В. Овсянников, Групповой анализ дифференииальных уравнений, Наука, М., 1978.

[8] Н. Х. Ибрагимов, Группь преобразований в математической физике, Наука, М., 1983.

[9] Р. К. Газизов, А. А. Касаткин, С. Ю. Лукащук, Вестн. УГАТУ, 9:3 (2007), 125-135.

[10] R. K. Gazizov, A. A. Kasatkin, S. Yu. Lukashchuk, Phys. Scr., 2009:T136 (2009), 014016, $5 \mathrm{pp}$.

[11] Р. К. Газизов, А.А. Касаткин, С. Ю. Лукащук, Уфимск. матем. журн., 4:4 (2012), $54-68$.

[12] E. Noether, Nachr. d. König. Gesellsch. d. Wiss. zu Göttingen, Math-phys. Klasse, 2 (1918), $235-257$.

[13] Н. Х. Ибрагимов, ТМФ, 1:3 (1969), 350-359.

[14] S.-H. Zhang, D.-Y. Chen, J.-L. Fu, Chinese Phys. B, 21:10 (2012), 100202, 8 pp.

[15] T. M. Atanackovic, S. Konjik, S. Pilipovic, S. Simic, Nonlinear Anal., 71:5-6 (2009), 1504-1517.

[16] G. S.F. Frederico, D. F. M. Torres, "Fractional Noether's theorem with classical and Riemann-Liouville derivatives", Decision and Control $(C D C)$, Proceedings of 51st IEEE Conference on Decision and Control (Maui, Hawaii, December 10-13, 2012), IEEE, New York, 2012, 6885-6890.

[17] G. S. F. Frederico, T. Odzijewicz, D. F. M. Torres, Appl. Anal., 96:1 (2014), 153-170.

[18] M. Klimek, J. Phys. A: Math. Theor., 34:31 (2001), 6167-6184.

[19] S. W. Wheatcraft, M. M. Meerschaert, Adv. Water Resour., 31:10 (2008), 1377-1381.

[20] V.E. Tarasov, Phys. Plasmas, 20:10 (2013), 102110, 10 pp., arXiv: 1307.4930.

[21] G.S. F. Frederico, D. F. M. Torres, J. Math. Anal. Appl., 334:2 (2007), 834-846.

[22] A. B. Malinowska, Appl. Math. Lett., 25:11 (2012), 1941-1946. 
[23] L. Bourdin, J. Cresson, I. Greff, Commun. Nonlinear Sci. Numer. Simulat., 18:4 (2013), $878-887$.

[24] N. H. Ibragimov, Elementary Lie Group Analysis and Ordinary Differential Equations, Wiley Series in Mathematical Methods in Practice, 4, John Wiley \& Sons, Chichester, 1999.

[25] Л.Э. Эльсгольц, Дифференциальные уравнения и вариачионное исчисление, Наука, М., 1969.

[26] O.P. Agrawal, J. Phys. A: Math. Theor., 40:21 (2007), 5469-5477.

[27] D. Baleanu, J. J. Trujillo, Nonlin. Dynam., 52:4 (2008), 331-335.

[28] P. Paradisi, R. Cesari, F. Mainardi, F. Tampieri, Physica A, 293:1-2 (2001), 130-142.

[29] D. A. Benson, S. W. Wheatcraft, M. M. Meerschaert, Water Resources Research, 36:6 (2000), 1403-1412.

[30] В. М. Головизнин, П. С. Кондратенко, Л. В. Матвеев, И. А. Короткин, И. Л. Драников, Аномальная дифбузия радионуклидов в сильнонеоднородных геологических формачияx, Наука, М., 2010.

[31] T. Odzijewicz, A. B. Malinowska, D. F. M. Torres, Europ. Phys. J., 222:8 (2013), 1813-1826.

[32] N. H. Ibragimov, J. Phys. A: Math. Theor., 44:43 (2011), 432002, 8 pp.

[33] Н. Х. Ибрагимов, Е. Д. Авдонина, УМН, 68:5(413) (2013), 111-146.

Поступила в редакцию 3.12 .2014 , после доработки 3.03.2015 